IU/NTC 97-07, TIFR/TH/97-38

\title{
Using Drell-Yan Processes to Probe Nucleon and Meson Structure Functions
}

\author{
R.S. Bhaleraot \\ Theoretical Physics Group \\ Tata Institute of Fundamental Research \\ Homi Bhabha Road, Colaba, Mumbai 400005 INDIA \\ J.T. Londergant \\ Department of Physics and Nuclear Theory Center \\ Indiana University \\ Bloomington IN 47408 USA
}

\begin{abstract}
We investigate how Drell-Yan processes can be used to measure the magnitude of flavor symmetry violation in the proton sea. We examine the utility of the following beams: protons, charged pions, and charged kaons. In each case we present an approximate expression for the Drell-Yan asymmetry. Using currently available parton distributions, we locate those kinematic regions which provide the greatest information on the quantity $\bar{d}^{p}(x)-\bar{u}^{p}(x)$. If sufficiently intense $K^{+}$beams were available, they could provide an efficient measurement of this quantity. Finally we present and discuss sets of sum rules for the Drell-Yan processes.
\end{abstract}

PACS: $13.85 . \mathrm{Q}, 25.80 . \mathrm{Nv}, 25.80 . \mathrm{Hp}, 11.30 . \mathrm{Hv}$

Typeset using REVTEX 


\section{INTRODUCTION}

At the present time there is considerable interest in the flavor structure of the nucleon sea [1]. This is largely a consequence of recent experimental results, such as the discovery by the New Muon Collaboration (NMC) of a violation of the Gottfried sum rule [2]. The most likely explanation of the NMC measurement was a significant flavor asymmetry in the proton sea, i.e. $\bar{d}^{p}(x) \neq \bar{u}^{p}(x)$. Although there is no compelling reason why one should have $\bar{d}^{p}(x)=\bar{u}^{p}(x)$, and in fact due to the Pauli principle one expects $\int_{0}^{1} d x\left[\bar{d}^{p}(x)-\bar{u}^{p}(x)\right]>0$, phenomenological fits to parton distributions prior to the NMC measurement had typically set these sea distributions equal.

Ellis and Stirling [3] pointed out that this asymmetry could be measured in protoninduced Drell-Yan (DY) processes on hydrogen and deuterium targets. They advocated measurements taken at center-of-mass rapidity $y=0$, i.e. $x_{1}=x_{2}$, where $x_{1}\left(x_{2}\right)$ is approximately the momentum fraction carried by the projectile (target) quark or antiquark which annihilates in the DY process. These measurements were carried out by the NA51 collaboration at CERN [4], which obtained the value

$$
\left.\frac{\bar{u}^{p}(x)}{\bar{d}^{p}(x)}\right|_{<x>=0.18}=0.51 \pm 0.04(\text { stat }) \pm 0.05(\text { syst }) \text {. }
$$

The $\bar{u}^{p} / \bar{d}^{p}$ distributions have recently been measured in the E866 experiment at FNAL [5], through proton-induced DY processes on hydrogen and deuterium. The E866 group has measured $\bar{u}^{p} / \bar{d}^{p}$ over a range of $x_{1}$ and $x_{2}$ values. With the Main Injector upgrade scheduled for completion in early 1999 at FNAL, there is a revival of interest in the physics issues which could be addressed with the beams of kaons that could become available. We think that there is interesting physics which could be done in that program.

In this paper we will review the prospects for measuring the flavor asymmetry in the nucleon sea through DY processes induced by protons or charged mesons $\left(K^{ \pm}\right.$and $\left.\pi^{ \pm}\right)$. We will examine the sensitivity of various cross sections to the up/down antiquark ratio in the proton. We will also look at the sensitivity to the kinematic region for both projectile and 
target. We will discuss what measurements are needed if the DY process is to be used to pin down the largely unknown kaon structure functions. Finally we will present and discuss sets of sum rules for the DY processes.

\section{ANALYTIC APPROXIMATIONS FOR DRELL-YAN ASYMMETRY}

The general form of the DY cross section is:

$$
\begin{aligned}
\frac{d^{2} \sigma^{D Y}}{d x_{1} d x_{2}} & =\left(\frac{4 \pi \alpha^{2}}{9 M^{2}}\right) \sum_{j} e_{j}^{2}\left[q_{j}^{B}\left(x_{1}\right) \bar{q}_{j}^{T}\left(x_{2}\right)+\bar{q}_{j}^{B}\left(x_{1}\right) q_{j}^{T}\left(x_{2}\right)\right] \\
& \equiv\left(\frac{4 \pi \alpha^{2}}{9 M^{2}}\right) H^{D Y}\left(x_{1}, x_{2}\right)
\end{aligned}
$$

In Eq. (2.1), $\alpha$ is the fine-structure constant, $M^{2}=s x_{1} x_{2}$ is the dilepton mass squared, $e_{j}$ is the charge of parton of flavor $j, q$ and $\bar{q}$ are the quark and antiquark distributions, and the superscripts $B$ and $T$ refer to the beam and target, respectively.

In order to probe differences between up and down antiquarks in the proton, one measures the ratio of DY cross sections on hydrogen and deuterium [4]. If one makes the impulse approximation, the DY cross section on deuterium $(D)$ will just equal the sum of DY cross sections on the free proton $(p)$ and neutron $(n)$. For a beam particle $B$ the asymmetry $A_{B}^{D Y} \equiv 2 \sigma_{B p} / \sigma_{B D}-1$ then becomes

$$
A_{B}^{D Y}\left(x_{1}, x_{2}\right)=\frac{H_{B p}^{D Y}\left(x_{1}, x_{2}\right)-H_{B n}^{D Y}\left(x_{1}, x_{2}\right)}{H_{B p}^{D Y}\left(x_{1}, x_{2}\right)+H_{B n}^{D Y}\left(x_{1}, x_{2}\right)}
$$

Because $A_{B}^{D Y}$ is a ratio of cross sections, one expects some systematic errors to cancel. In subsequent sections, we will suppress for simplicity the dependence of $A_{B}^{D Y}$ on $x_{1}$ and $x_{2}$.

In comparing DY cross sections on protons and neutrons, we invoke charge symmetry for the nucleon parton distributions. This involves, for example, setting

$$
u^{n}(x)=d^{p}(x) \text { and } d^{n}(x)=u^{p}(x)
$$

and similarly for the antiquark distributions. We can therefore define all nucleon parton distributions in terms of those in the proton. Charge symmetry violating [CSV] effects have 
been estimated by Londergan, Thomas and collaborators [6,7]. For these processes the CSV effects should never be greater than $1 \%$ of the relevant amplitudes.

Contributions to $A_{B}^{D Y}$ which involve an up quark from the projectile will then be directly proportional to $\bar{u}^{p}(x)-\bar{d}^{p}(x)$. Since up quark terms will be four times as large as down quark terms, due to the square of the quark charge, such terms should make substantial contributions to the measured asymmetries. For this reason $A_{B}^{D Y}$ should be an excellent place to measure up/down differences in the proton sea. This does not mean that all projectiles would be equally good probes of $\bar{u}^{p}(x)-\bar{d}^{p}(x)$. In fact $p$ and $K^{+}$turn out to be better than $K^{-}, \pi^{+}$and $\pi^{-}$. Reasons for this are discussed in section IV.

In subsequent sections, we make the following approximations. We neglect nuclear cor-

rections to free structure functions in deuterium [8]. We neglect heavy-quark (charm and heavier) contributions. We assume $q_{\text {sea }}(x)=\bar{q}_{\text {sea }}(x)$. Finally, while displaying various equations we ignore sea-sea contributions; these will be included in actual calculations, but are neglected here for simplicity.

\section{A. Drell-Yan Asymmetry for Proton Beams}

For proton projectile we have

$$
H_{p p}^{D Y}\left(x_{1}, x_{2}\right)=\frac{1}{9}\left[4\left(u_{v}^{p}\left(x_{1}\right) \bar{u}_{s}^{p}\left(x_{2}\right)+\bar{u}_{s}^{p}\left(x_{1}\right) u_{v}^{p}\left(x_{2}\right)\right)+d_{v}^{p}\left(x_{1}\right) \bar{d}_{s}^{p}\left(x_{2}\right)+\bar{d}_{s}^{p}\left(x_{1}\right) d_{v}^{p}\left(x_{2}\right)\right],
$$

and a similar expression for $H_{p n}^{D Y}\left(x_{1}, x_{2}\right)$. The asymmetry is

$$
\begin{aligned}
A_{p}^{D Y} & =\frac{H_{p p}^{D Y}-H_{p n}^{D Y}}{H_{p p}^{D Y}+H_{p n}^{D Y}} \\
& =\frac{-\left(4 u_{v}^{p}\left(x_{1}\right)-d_{v}^{p}\left(x_{1}\right)\right)\left(\bar{d}_{s}^{p}\left(x_{2}\right)-\bar{u}_{s}^{p}\left(x_{2}\right)\right)+\left(4 \bar{u}_{s}^{p}\left(x_{1}\right)-\bar{d}_{s}^{p}\left(x_{1}\right)\right)\left(u_{v}^{p}\left(x_{2}\right)-d_{v}^{p}\left(x_{2}\right)\right)}{\left(4 u_{v}^{p}\left(x_{1}\right)+d_{v}^{p}\left(x_{1}\right)\right)\left(\bar{d}_{s}^{p}\left(x_{2}\right)+\bar{u}_{s}^{p}\left(x_{2}\right)\right)+\left(4 \bar{u}_{s}^{p}\left(x_{1}\right)+\bar{d}_{s}^{p}\left(x_{1}\right)\right)\left(u_{v}^{p}\left(x_{2}\right)+d_{v}^{p}\left(x_{2}\right)\right)} .
\end{aligned}
$$

In Fig. 1, we show the asymmetry $A_{p}^{D Y}\left(x_{1}=x_{2}\right)$ vs. $x_{1}$. Recall that $x_{1} \equiv x_{\text {beam }}$ and $x_{2} \equiv$ $x_{\text {target }}$. The curves are obtained with the phenomenological nucleon parton distributions of Glück, Reya and Vogt [9] with $Q^{2}=20 \mathrm{GeV}^{2}$. (Calculations were also performed with CTEQ-3M [10] and $\operatorname{MRS}(\mathrm{G})$ [11] parton distributions; results were similar and are not 
shown.) The lower curve is obtained with the full parton distributions, while the upper one results from fixing $\bar{u}^{p}(x)=\bar{d}^{p}(x)$. For this kinematics, the two asymmetries have opposite signs: If we allow flavor asymmetry, the predicted DY asymmetry is negative, while it is positive if we require $\mathrm{SU}(2)$ flavor symmetry. In some kinematic regions the asymmetry $A_{p}^{D Y}$ is large, but the DY cross sections may be so small that it cannot be accurately measured.

Figure 1 requires that we measure the DY asymmetry along the line $x_{1}=x_{2}$. We can examine whether this is the most sensitive region to test flavor asymmetry in the nucleon sea. In Fig. 2, we show how the asymmetry $A_{p}^{D Y}$ varies with $x_{1}$ and $x_{2}$. In Fig. 2a, we plot equal- $A_{p}^{D Y}$ contours. Here and in other figures, the two extreme contours are labelled; the intermediate contours are understood to have labels differing in steps of 0.1. In Fig. 20 b, we plot the equal- $A_{p}^{D Y}$ contours obtained by fixing $\bar{u}^{p}(x)=\bar{d}^{p}(x)$. In Fig. 2 $1 \mathrm{c}$ we show contours of equal asymmetry difference $\Delta_{p}^{D Y}$ defined by

$$
\left.\Delta_{p}^{D Y}\left(x_{1}, x_{2}\right) \equiv A_{p}^{D Y}\right|_{\bar{u}=\bar{d}}-\left.A_{p}^{D Y}\right|_{\bar{u} \neq \bar{d}}
$$

Along the line $x_{1}=x_{2}$ (the dashed curve in Fig. 2a), we see that the asymmetry $A_{p}^{D Y}$ is negative and becomes more negative with increasing $x$. Secondly, if we set $\bar{u}^{p}(x)=\bar{d}^{p}(x)$ and $x_{1}=x_{2}$, the resulting asymmetry is positive and roughly constant at $A_{p}^{D Y} \approx 0.1-0.2$; see Fig. 2b. Finally, the difference between the two asymmetries goes on increasing as $x_{1}=x_{2}$ increases; see Fig. 2c. All these features are consistent with Fig. 1. Thus the equal- $x$ kinematics is quite adequate if the purpose is to maximize the difference $\Delta_{p}^{D Y}$.

However, if the purpose is to maximize the asymmetry $A_{p}^{D Y}$, the equal-x kinematics does not seem to be the optimum choice. The asymmetry can be significantly altered by deviating from the dotted line in Fig. 2a. If $x_{1}=x_{2}=0.2$, the asymmetry is about $-10 \%$ which is consistent with the experimental observation in Ref. [4]. However, if the measurements had been made at $x_{1}=0.3$ and $x_{2}=0.2$, the asymmetry would have been $\approx-20 \%$. On the other hand, if the measurements had been made at $x_{1}=0.1$ and $x_{2}=0.2$, the asymmetry would have been nearly zero. Experimentally the ratio $\left(\sigma_{B D} / 2 \sigma_{B p}\right)$ is measured, and then the asymmetry $A_{p}^{D Y}$ is deduced. Contours for a fixed $\left(\sigma_{B D} / 2 \sigma_{B p}\right)$ are identical to those for 
a fixed $A_{p}^{D Y}$ (Fig. 2a); only the contours labels are different.

The E866 group at FNAL [5] has measured DY processes for proton projectiles on protons and deuterons over a wide kinematic region. They focus especially on the region of large $x_{1} \equiv$ $x_{\text {beam }}$ and small $x_{2} \equiv x_{\text {target }}$. For sufficiently large $x_{1}$, the contribution from the projectile sea in Eq. 2.4 is negligible relative to the valence contribution. Since $d_{v}^{p}\left(x_{1}\right) / u_{v}^{p}\left(x_{1}\right)<1$, in this kinematic region the ratio of Drell-Yan cross sections is approximately given by

$$
\frac{\sigma_{p D}}{2 \sigma_{p p}}=\left[1+A_{p}^{D Y}\right]^{-1} \approx \frac{1}{2}\left[1+\frac{\bar{d}^{p}\left(x_{2}\right)}{\bar{u}^{p}\left(x_{2}\right)}\right],
$$

as can be seen from Eq. 2.5. The E866 group is thus able to measure the ratio $\bar{d}^{p} / \bar{u}^{p}$ over a wide range of $x$ [5].

\section{B. Drell-Yan Asymmetry for Kaon ${ }^{ \pm}$Beams}

For $K^{+}$projectile we have

$$
H_{K^{+} p}^{D Y}=\frac{1}{9}\left[4\left(u_{v}^{K^{+}} \bar{u}_{s}^{p}+\bar{u}_{s}^{K^{+}} u_{v}^{p}\right)+\bar{d}_{s}^{K^{+}} d_{v}^{p}+\bar{s}_{v}^{K^{+}} s_{s}^{p}\right]
$$

and a similar expression for $H_{K^{+}{ }^{D}}^{D Y}$. Here we have suppressed for convenience the dependence of the parton distributions on $x_{1}$ or $x_{2}$, since there is no ambiguity in this case. The asymmetry is

$$
\begin{aligned}
A_{K^{+}}^{D Y} & =\frac{H_{K^{+} p}^{D Y}-H_{K^{+} n}^{D Y}}{H_{K^{+} p}^{D Y}+H_{K^{+}}^{D Y}} \\
& =\frac{-4 u_{v}^{K^{+}}\left(\bar{d}_{s}^{p}-\bar{u}_{s}^{p}\right)+\left(4 \bar{u}_{s}^{K^{+}}-\bar{d}_{s}^{K^{+}}\right)\left(u_{v}^{p}-d_{v}^{p}\right)}{4 u_{v}^{K^{+}}\left(\bar{d}_{s}^{p}+\bar{u}_{s}^{p}\right)+\left(4 \bar{u}_{s}^{K^{+}}+\bar{d}_{s}^{K^{+}}\right)\left(u_{v}^{p}+d_{v}^{p}\right)+2 \bar{s}_{v}^{K^{+}} s_{s}^{p}} .
\end{aligned}
$$

In Fig. 3 we show the asymmetry $A_{K^{+}}^{D Y}\left(x_{1}=x_{2}\right)$ vs. $x_{1}$. The curves use the GRV parton distributions for the nucleons [9]. Since little experimental information is available on kaon structure functions, we use in their place the GRV distributions for the pion [9]. The lower curve uses the full parton distributions, while the upper curve is obtained if we fix $\bar{u}^{p}(x)=\bar{d}^{p}(x)$. Note that for $x$ between 0.1 and 0.4 , the difference between the two 
asymmetries is quite large. If one had an intense well separated $K^{+}$beam, it would, in principle, be an excellent probe of flavor asymmetry in the proton sea.

As is clear from Eq. 2.9, in order to calculate $A_{K^{+}}^{D Y}$ we need four different parton distribution functions for $\mathrm{K}^{+}$, while the only experimental information we have is on the ratio $\bar{u}^{K^{-}} / \bar{u}^{\pi^{-}}$[12]. This ratio was found to be consistent with unity for $x_{1} \leq 0.7$, and was less than unity only for larger values of $x_{1}$. It was determined by using an unseparated negative beam containing pions, kaons and antiprotons, and by making the following assumptions: the meson sea was neglected, among the valence quarks in the mesons only the up flavor was retained, and finally the Drell-Yan scale factors for $K^{-}$and $\pi^{-}$were assumed to be identical and constant over the kinematic range explored. This points out the urgent need for more experimental data on kaon structure functions, and justifies our use of pion structure functions in place of kaon structure functions in the calculation of the Drell-Yan asymmetry.

We again examine whether the region of equal- $x$ kinematics is the most sensitive region to probe the flavor asymmetry in the proton sea. Figures 4 a-c correspond to Figs. 2 a-c, respectively, with the projectile proton replaced by a $K^{+}$projectile. In the case of the proton projectile, we saw that the equal- $x$ kinematics was adequate for the purpose of maximizing the difference $\Delta_{p}^{D Y}$ (Fig. 2c), but was not optimum for the purpose of maximizing the asymmetry $A_{p}^{D Y}$ (Fig. 2a). In the case of the $K^{+}$projectile, we find that the equal-x kinematics is not good for either purpose (Figs. 4a and 4c). It is clear from Fig. 4a that if $x_{1}=x_{2}<0.3$ the asymmetry is nearly zero, but can be significantly enhanced by deviating from the $x_{1}=x_{2}$ line.

The asymmetry for $\mathrm{K}^{-}$mesons incident on protons and neutrons (in the deuterium) can be calculated similarly. Using the same approximations as before, we obtain

$$
H_{K^{-} p}^{D Y}=\frac{1}{9}\left[4\left(\bar{u}_{v}^{K^{-}} u_{v}^{p}+\bar{u}_{s}^{K^{-}} u_{v}^{p}+\bar{u}_{v}^{K^{-}} u_{s}^{p}\right)+\bar{d}_{s}^{K^{-}} d_{v}^{p}+s_{v}^{K^{-}} \bar{s}_{s}^{p}\right]
$$

and a similar expression for $H_{K^{-} n}^{D Y}$. The asymmetry is

$$
A_{K^{-}}^{D Y}=\frac{H_{K^{-} p}^{D Y}-H_{K^{-} n}^{D Y}}{H_{K^{-} p}^{D Y}+H_{K^{-} n}^{D Y}}
$$




$$
=\frac{-4 \bar{u}_{v}^{K^{-}}\left(\bar{d}_{s}^{p}-\bar{u}_{s}^{p}\right)+\left(4 \bar{u}_{v}^{K^{-}}+4 \bar{u}_{s}^{K^{-}}-\bar{d}_{s}^{K^{-}}\right)\left(u_{v}^{p}-d_{v}^{p}\right)}{4 \bar{u}_{v}^{K^{-}}\left(\bar{d}_{s}^{p}+\bar{u}_{s}^{p}\right)+\left(4 \bar{u}_{v}^{K^{-}}+4 \bar{u}_{s}^{K^{-}}+\bar{d}_{s}^{K^{-}}\right)\left(u_{v}^{p}+d_{v}^{p}\right)+2 s_{v}^{K^{-}} \bar{s}_{s}^{p}} .
$$

Numerical calculations show that this asymmetry is insensitive to the up/down difference in the proton sea-quark distributions (see section IV).

\section{Drell-Yan Asymmetry for Pion ${ }^{ \pm}$Beams}

For $\pi^{+}$projectile we have

$$
H_{\pi^{+} p}^{D Y}=\frac{1}{9}\left[4\left(u_{v}^{\pi^{+}} \bar{u}_{s}^{p}+\bar{u}_{s}^{\pi^{+}} u_{v}^{p}\right)+\bar{d}_{v}^{\pi^{+}} d_{v}^{p}+\bar{d}_{v}^{\pi^{+}} d_{s}^{p}+\bar{d}_{s}^{\pi^{+}} d_{v}^{p}\right]
$$

and a similar expression for $H_{\pi^{+} n}^{D Y}$. The asymmetry is

$$
\begin{aligned}
A_{\pi^{+}}^{D Y} & =\frac{H_{\pi^{+} p}^{D Y}-H_{\pi^{+} n}^{D Y}}{H_{\pi^{+} p}^{D Y}+H_{\pi^{+} n}^{D Y}} \\
& =\frac{-\left(4 u_{v}^{\pi^{+}}-\bar{d}_{v}^{\pi^{+}}\right)\left(\bar{d}_{s}^{p}-\bar{u}_{s}^{p}\right)+\left(4 \bar{u}_{s}^{\pi^{+}}-\bar{d}_{v}^{\pi^{+}}-\bar{d}_{s}^{\pi^{+}}\right)\left(u_{v}^{p}-d_{v}^{p}\right)}{\left(4 u_{v}^{\pi^{+}}+\bar{d}_{v}^{\pi^{+}}\right)\left(\bar{d}_{s}^{p}+\bar{u}_{s}^{p}\right)+\left(4 \bar{u}_{s}^{\pi^{+}}+\bar{d}_{v}^{\pi^{+}}+\bar{d}_{s}^{\pi^{+}}\right)\left(u_{v}^{p}+d_{v}^{p}\right)} .
\end{aligned}
$$

For $\pi^{-}$beams, one obtains

$$
H_{\pi^{-} p}^{D Y}=\frac{1}{9}\left[4\left(\bar{u}_{v}^{\pi^{-}} u_{v}^{p}+\bar{u}_{s}^{\pi^{-}} u_{v}^{p}+\bar{u}_{v}^{\pi^{-}} u_{s}^{p}\right)+d_{v}^{\pi^{-}} \bar{d}_{s}^{p}+\bar{d}_{s}^{\pi^{-}} d_{v}^{p}\right]
$$

and a similar expression for $H_{\pi^{-} n}^{D Y}$. The asymmetry is

$$
\begin{aligned}
A_{\pi^{-}}^{D Y} & =\frac{H_{\pi^{-} p}^{D Y}-H_{\pi^{-} n}^{D Y}}{H_{\pi^{-}}^{D Y}+H_{\pi^{-}}^{D Y}} \\
& =\frac{-\left(4 \bar{u}_{v}^{\pi^{-}}-d_{v}^{\pi^{-}}\right)\left(\bar{d}_{s}^{p}-\bar{u}_{s}^{p}\right)+\left(4 \bar{u}_{v}^{\pi^{-}}+4 \bar{u}_{s}^{\pi^{-}}-\bar{d}_{s}^{\pi^{-}}\right)\left(u_{v}^{p}-d_{v}^{p}\right)}{\left(4 \bar{u}_{v}^{\pi^{-}}+d_{v}^{\pi^{-}}\right)\left(\bar{d}_{s}^{p}+\bar{u}_{s}^{p}\right)+\left(4 \bar{u}_{v}^{\pi^{-}}+4 \bar{u}_{s}^{\pi^{-}}+\bar{d}_{s}^{\pi^{-}}\right)\left(u_{v}^{p}+d_{v}^{p}\right)} .
\end{aligned}
$$

Numerical calculations show that both $A_{\pi^{+}}^{D Y}$ and $A_{\pi^{-}}^{D Y}$ are insensitive to the up/down difference in the proton sea-quark distributions (see section IV).

\section{DRELL-YAN SUM RULES}

If one assumes (a) charge conjugation symmetry so that e.g., $q^{\pi^{+}}=\bar{q}^{\pi^{-}}$, (b) isospin symmetry so that e.g., $u^{\pi^{+}}=d^{\pi^{-}}$, (c) $q_{\text {sea }}(x)=\bar{q}_{\text {sea }}(x)$, and (d) that charm and heavier 
flavors are negligible, then it is straightforward to show that $\pi^{+}$has only three chargedparton distribution functions (PDF), namely

$$
\begin{aligned}
& u_{\text {val }}(x)=\bar{d}_{\text {val }}(x) \equiv V^{\pi}(x), \\
& u_{\text {sea }}(x)=\bar{u}_{\text {sea }}(x)=d_{\text {sea }}(x)=\bar{d}_{\text {sea }}(x) \equiv S_{\text {light }}^{\pi}(x), \\
& s_{\text {sea }}(x)=\bar{s}_{\text {sea }}(x) \equiv S_{\text {heavy }}^{\pi}(x),
\end{aligned}
$$

and those for $\pi^{-}$can be identified with one of these three. Thus $\pi^{+}$and $\pi^{-}$together have only three charged-parton distribution functions. With the same set of assumptions (a)(d), it is easy to show that $K^{+}, K^{-}, K^{0}$ and $\bar{K}^{0}$ together have only five charged-parton distribution functions which we denote by

$$
V_{\text {light }}^{K}(x), \quad V_{\text {heavy }}^{K}(x), \quad S_{\text {light }, 1}^{K}(x), \quad S_{\text {light }, 2}^{K}(x), \quad S_{\text {heavy }}^{K}(x),
$$

where the notation is as in Eq. (3.1).

To experimentally determine the three PDF's for pions, Eq. (3.1), one needs data on any three of the following four quantities:

$$
H_{\pi^{-} D}^{D Y}, \quad H_{\pi^{-} p}^{D Y}, \quad H_{\pi^{+} D}^{D Y}, \quad H_{\pi^{+} p}^{D Y}
$$

Out of these, the first three may be preferable due to higher event rates. In fact, if the sea-sea contribution to $H_{\pi}^{D Y}$ is ignored then $H_{\pi}^{D Y}$ would contain only $V^{\pi}(x)$ and $S_{\text {light }}^{\pi}(x)$, and data on only two of the four quantities in (3.3) would be sufficient.

Similarly, if the sea-sea contribution to $H_{K}^{D Y}$ is ignored, then $S_{\text {heavy }}^{K}(x)$ does not occur in $H_{K}^{D Y}$. To determine the remaining four PDF's for $K$, one needs data on

$$
H_{K^{-} D}^{D Y}, \quad H_{K^{-} p}^{D Y}, \quad H_{K^{+} D}^{D Y}, \quad H_{K^{+} p}^{D Y} .
$$

If further the difference between $S_{\text {light }, 1}^{K}$ and $S_{\text {light, } 2}^{K}$ is ignored, then the data on any three quantities in (3.4) would be sufficient.

As an alternative, some of these PDF's can be determined with the help of DY sum rules which we now present. For a beam particle $B$ incident on the deuterium $(D)$ target, 
we assume $H_{B D}^{D Y}=H_{B p}^{D Y}+H_{B n}^{D Y}$. Recall also that $x_{1} \equiv x_{\text {beam }}$ and $x_{2} \equiv x_{\text {target }}$. It is straightforward to show that

$$
\begin{gathered}
\int\left(H_{\pi^{-} p}^{D Y}-H_{\pi^{+} p}^{D Y}\right) d x_{2}=7 / 9 \cdot V^{\pi}\left(x_{1}\right), \\
\int\left(H_{\pi^{-} D}^{D Y}-H_{\pi^{+} D}^{D Y}\right) d x_{2}=V^{\pi}\left(x_{1}\right), \\
\int\left(H_{K^{-} p}^{D Y}-H_{K^{+} p}^{D Y}\right) d x_{2}=8 / 9 \cdot V_{\text {light }}^{K}\left(x_{1}\right), \\
\int\left(H_{K^{-} D}^{D Y}-H_{K^{+} D}^{D Y}\right) d x_{2}=4 / 3 \cdot V_{\text {light }}^{K}\left(x_{1}\right),
\end{gathered}
$$

where the limits of integration are 0 and 1 . Note the interesting fact that these rules do not require any knowledge of the target particle structure functions. If the integration was done over $x_{1}$ instead of $x_{2}$, we would have got a different set of sum rules with the well known nucleon structure functions, instead of the poorly known meson structure functions, occurring on the right-hand sides of Eqs. (3.5).

There are additional sum rules where the integration is done over both $x_{1}$ and $x_{2}$ :

$$
\begin{gathered}
\iint\left(H_{\bar{p} p}^{D Y}-H_{p p}^{D Y}\right) d x_{1} d x_{2}=17 / 9 \\
\iint\left(H_{\bar{p} D}^{D Y}-H_{p D}^{D Y}\right) d x_{1} d x_{2}=3 \\
\iint\left(H_{K^{-} p}^{D Y}-H_{K^{+} p}^{D Y}\right) d x_{1} d x_{2}=8 / 9 \\
\iint\left(H_{K^{-} D}^{D Y}-H_{K^{+} D}^{D Y}\right) d x_{1} d x_{2}=4 / 3 \\
\iint\left(H_{\pi^{-} p}^{D Y}-H_{\pi^{+} p}^{D Y}\right) d x_{1} d x_{2}=7 / 9 \\
\iint\left(H_{\pi^{-} D}^{D Y}-H_{\pi^{+} D}^{D Y}\right) d x_{1} d x_{2}=1 .
\end{gathered}
$$

If QCD corrections are included, all the right-hand sides in the above sum rules will get multiplied by the well known DY $K$ factor whose value is about 2. Since the nucleon PDF's are rather well known, one may also look at the first of Eqs. (3.6) as a way to determine the average DY $K$ factor. It may not be easy to verify these sum rules experimentally, because each rule involves two experiments (e.g., with $\pi^{-}$and $\pi^{+}$beams). Moreover, the integration is over the entire kinematic range which would entail some extrapolation of the data. However, if the beams do not have great intensity, the integration may help in getting 
enough counts to make a measurement. Thus, if suitable beams are available, experimental verification may not be impossible. Sum rules similar to those in Eq. (3.6) were given by Hwa 13]. The fact that the valence contributions to the DY process can be isolated by taking the difference between particle beam and antiparticle beam on a given target was mentioned by Sarma [14], but no sum rule was given.

\section{CONCLUSIONS}

Why are the proton and $K^{+}$projectiles more sensitive than the $K^{-}, \pi^{+}$and $\pi^{-}$projectiles, to the flavor asymmetry in the proton sea? Inspection of the expressions for $A_{B}^{D Y}$ for various projectiles shows that they all contain a contribution proportional to $\left(\bar{d}_{s}^{p}\left(x_{2}\right)-\bar{u}_{s}^{p}\left(x_{2}\right)\right)$ and a contribution proportional to $\left(u_{v}^{p}\left(x_{2}\right)-d_{v}^{p}\left(x_{2}\right)\right)$. The former constitutes the "signal" while the latter is "noise". For all five projectiles, the signal is getting multiplied by valence parton distribution functions in the projectile. But these projectiles differ from each other in the following respect. In the case of $p$ and $K^{+}$projectiles, the noise is getting multiplied by the sea parton distribution functions from the projectile, while for the $K^{-}, \pi^{+}$and $\pi^{-}$ projectiles, the noise is getting multiplied by valence parton distribution functions from the projectile. Naturally in the latter case, the signal is expected to be relatively weak. This is borne out by the numerical calculations presented here. The difference between the numerical results for $p$ and $K^{+}$projectiles on one hand and the $K^{-}, \pi^{+}$and $\pi^{-}$projectiles on the other, can be traced to the fact that $p$ and $K^{+}$contain neither $\bar{u}$ nor $\bar{d}$ as valence partons, while $K^{-}, \pi^{+}$and $\pi^{-}$do.

Results presented in Figs. 2 and 4 bring into focus the kinematic regions which can be optimally explored in future experiments designed to probe the flavor asymmetry in the proton sea. Finally we have presented sets of Drell-Yan sum rules; those in Eq. (3.5), for example, could throw some light on the poorly known meson structure functions. The first of Eqs. (3.6) could be used to deduce the DY $K$ factor averaged over the full kinematic region. 


\section{ACKNOWLEDGMENTS}

One of the authors [RSB] thanks the Indiana University Nuclear Theory Center for its hospitality during the period this work was undertaken. One of the authors [JTL] was supported in part by the US NSF under research contracts NSF-PHY-9408843 and NSFPHY-9722076. RSB thanks the late Prof. K.V.L. Sarma for many useful discussions and comments on the manuscript. 


\section{REFERENCES}

* e-mail: bhalerao@theory.tifr.res.in

$\dagger \quad$ e-mail: tlonderg@iucf.indiana.edu

[1] see, e.g., S. Kumano, Flavor Asymmetry of Antiquark Distributions in the Nucleon, preprint no. SAGA-HE-97-97, e-print hep-ph/9702367, submitted to Phys. Rep.

[2] New Muon Collaboration, P. Amaudruz et al. Phys. Rev. Lett. 66, 2712 (1991); M. Arneodo et al. Phys. Rev. D50, R1 (1994).

[3] S. D. Ellis and W. J. Stirling, Phys. Lett. B256, 258 (1991).

[4] NA51 Collaboration, A. Baldit et al. Phys. Lett. B332, 244 (1994).

[5] E866 Collaboration, E. Hawker et al., Proceedings of XXXII Moriond Conference, Mar. 1997 (to be published); P. Reimer et al., Proceedings of Sixth Conference on the Intersections of Particle and Nuclear Physics, May 1997 (to be published).

[6] E. N. Rodionov, A. W. Thomas and J. T. Londergan, Mod. Phys. Lett. A9, 1799 (1994).

[7] J. T. Londergan, G. T. Garvey, G. Q. Liu, E. N. Rodionov and A. W. Thomas, Phys. Lett. B340, 115 (1994).

[8] W. Melnitchouk and A. W. Thomas, Phys. Rev. D47, 3783 (1993).

[9] M. Glück, E. Reya and A. Vogt, Z. Phys. C67, 433 (1995); C53, 651 (1992).

[10] H. L. Lai et al., Phys. Rev. D51, 4763 (1995).

[11] A. D. Martin, W. J. Stirling and R. G. Roberts, Phys. Lett. B354, 155 (1995).

[12] NA3 Collaboration, J. Badier et al. Phys. Lett. B93, 354 (1980).

[13] R. C. Hwa, Phys. Rev. Lett. 40, 1218 (1978).

[14] K. V. L. Sarma, Phys. Rev. D22, 216 (1980). 


\section{FIGURES}

FIG. 1. Theoretical Drell-Yan asymmetry $A_{p}^{D Y}\left(x_{1}=x_{2}\right)$ vs. $x_{1}$ for proton beam; see Eq. (2.5). Here $x_{1} \equiv x_{\text {beam }}$ and $x_{2} \equiv x_{\text {target }}$. The lower curve is calculated with full parton distributions, the upper one fixing $\bar{u}^{p}(x)=\bar{d}^{p}(x)$.

FIG. 2. Contour plots of Drell-Yan asymmetries for the proton beam. (a) Contours of equal asymmetry $A_{p}^{D Y}$, using full GRV parton distributions of Ref. [9]. (b) Contours of equal asymmetry using GRV parton distributions but fixing $\bar{u}^{p}(x)=\bar{d}^{p}(x)$. (c) Contours of equal difference $\Delta_{p}^{D Y}$, defined in Eq. (2.6). The two extreme contours are labelled; the intermediate contours are understood to have labels differing in steps of 0.1. Dashed curve: $x_{\text {beam }}=x_{\text {target }}$.

FIG. 3. Same as Fig. 1 except that the proton beam is replaced by the $K^{+}$beam.

FIG. 4. Same as Fig. 2 except that the proton beam is replaced by the $K^{+}$beam. 


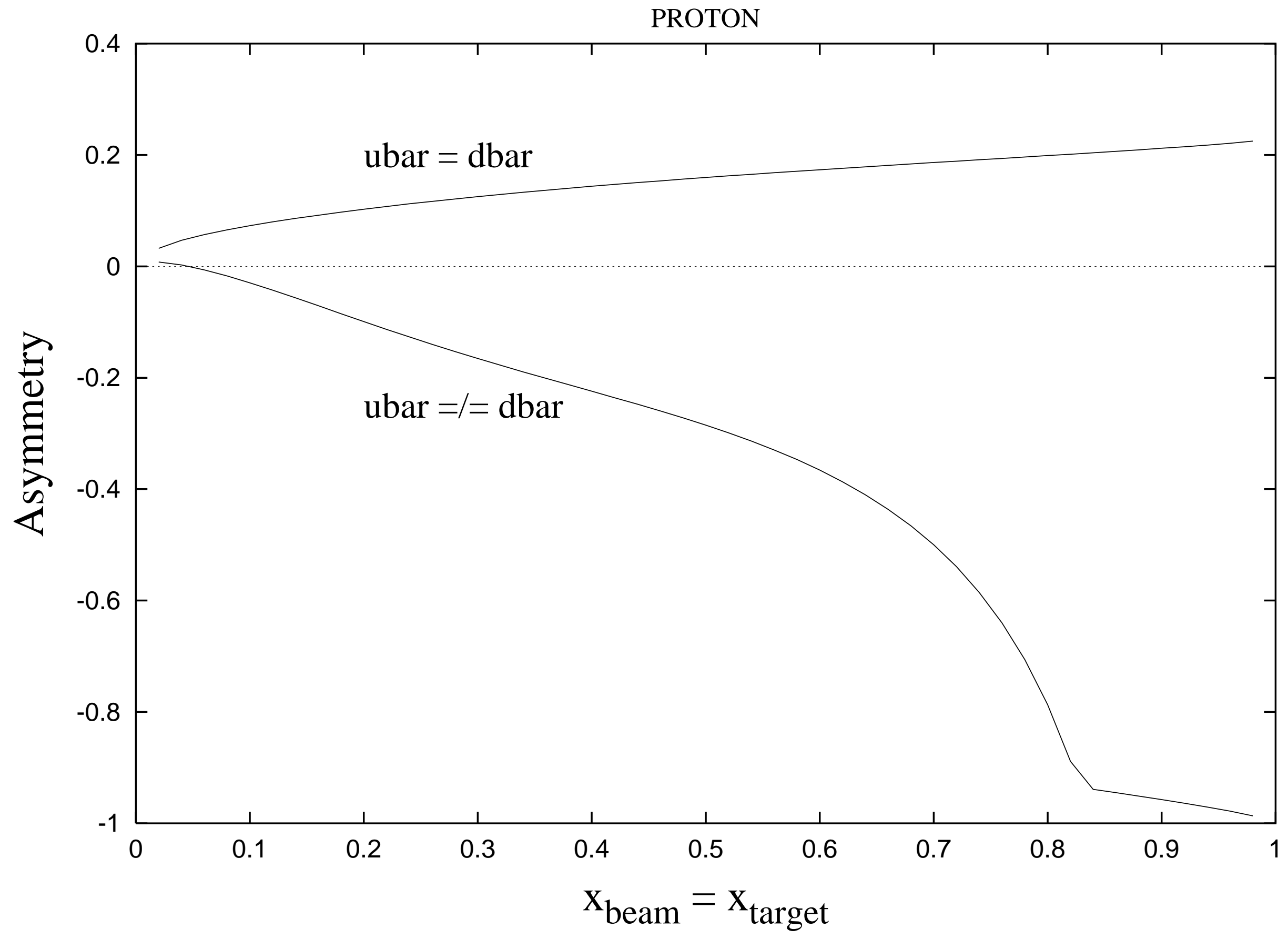




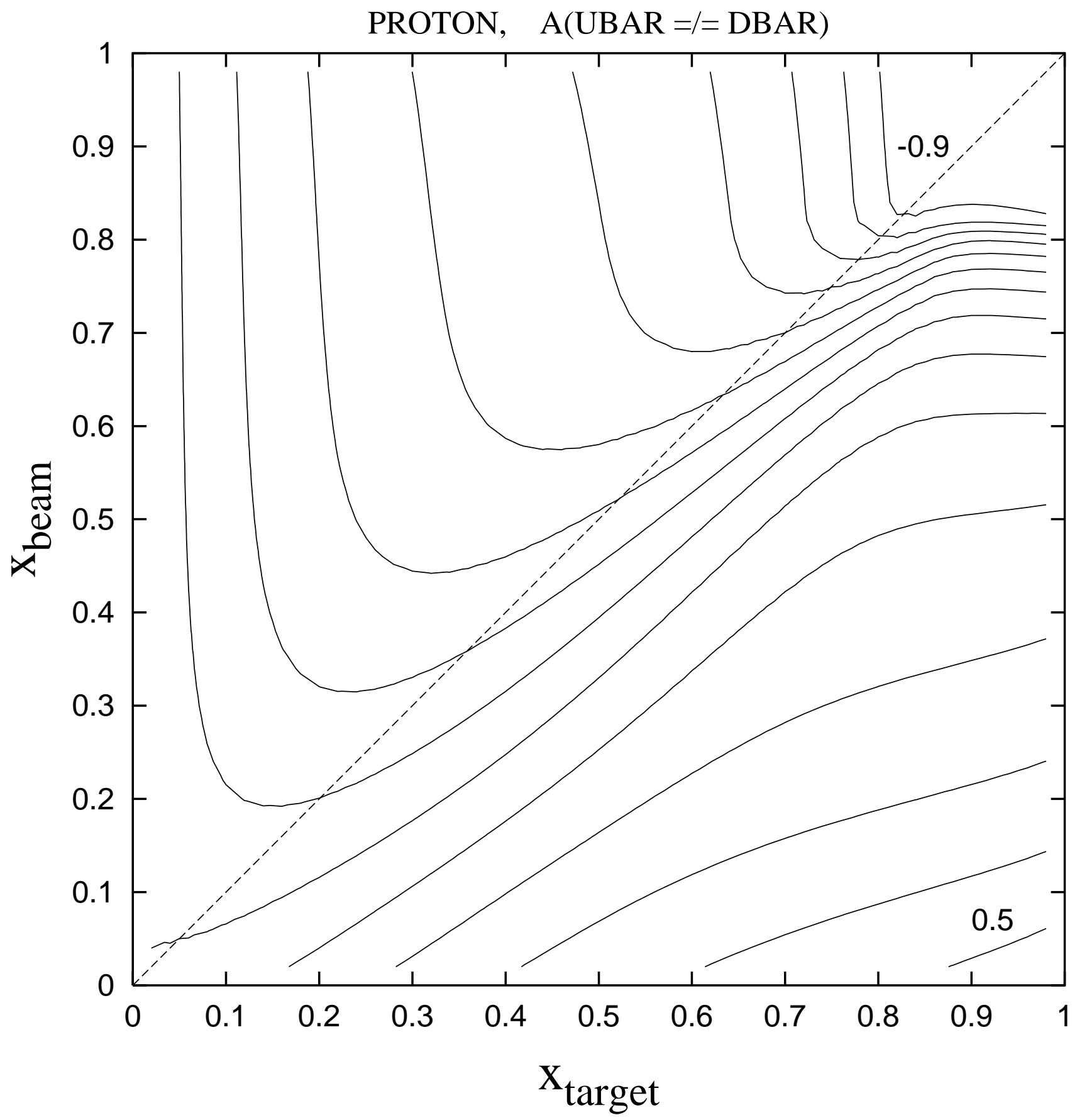




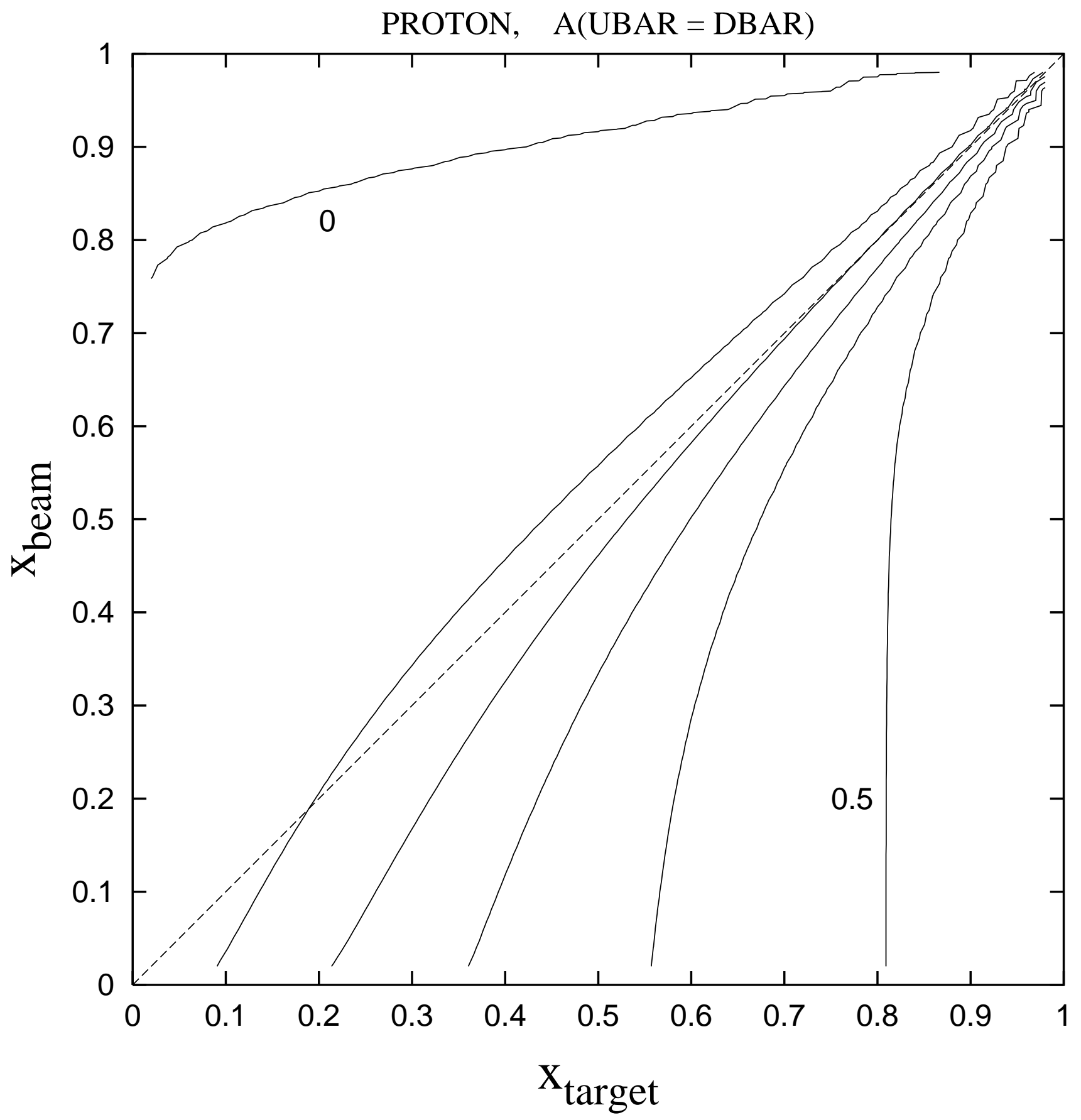




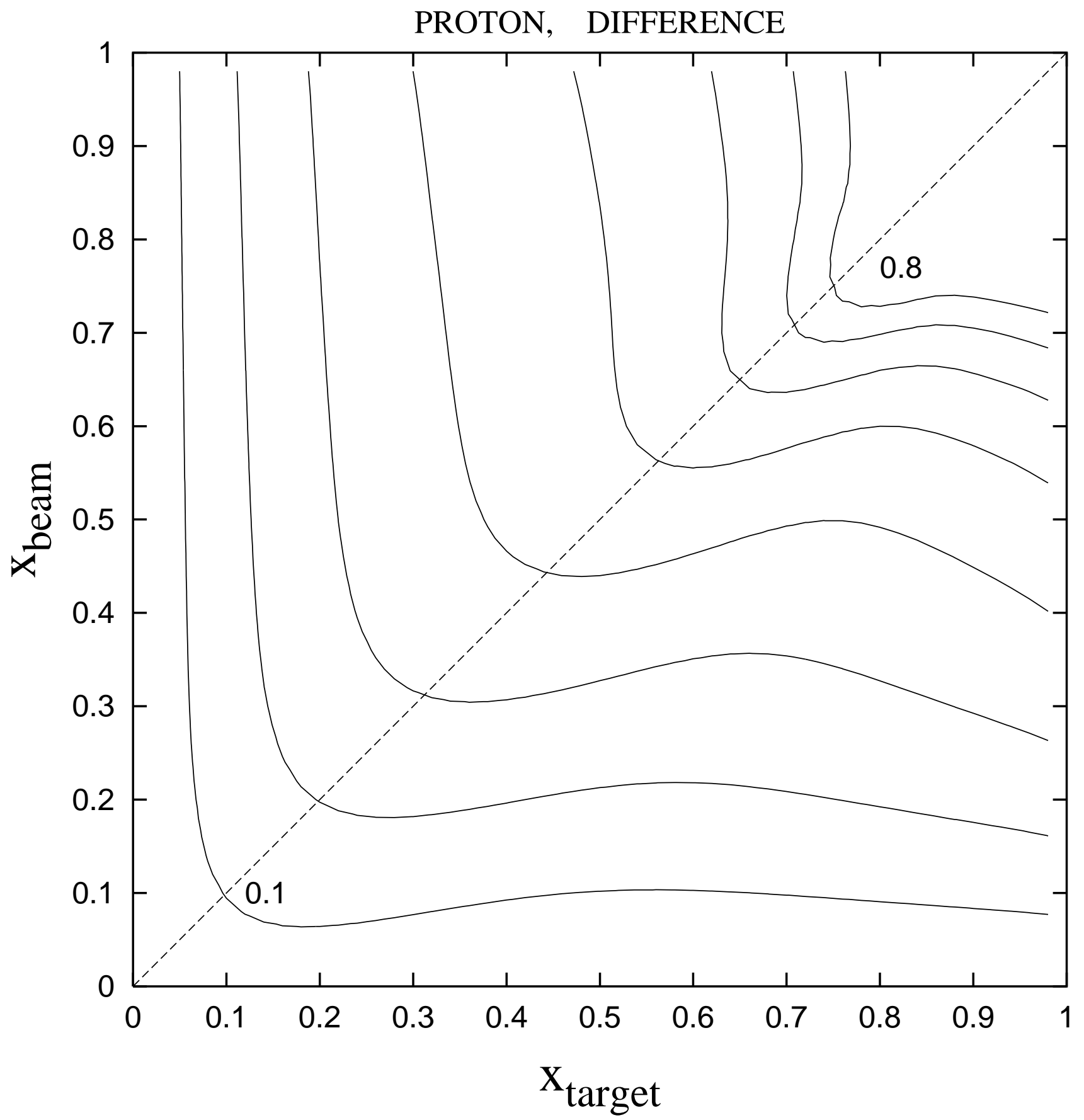




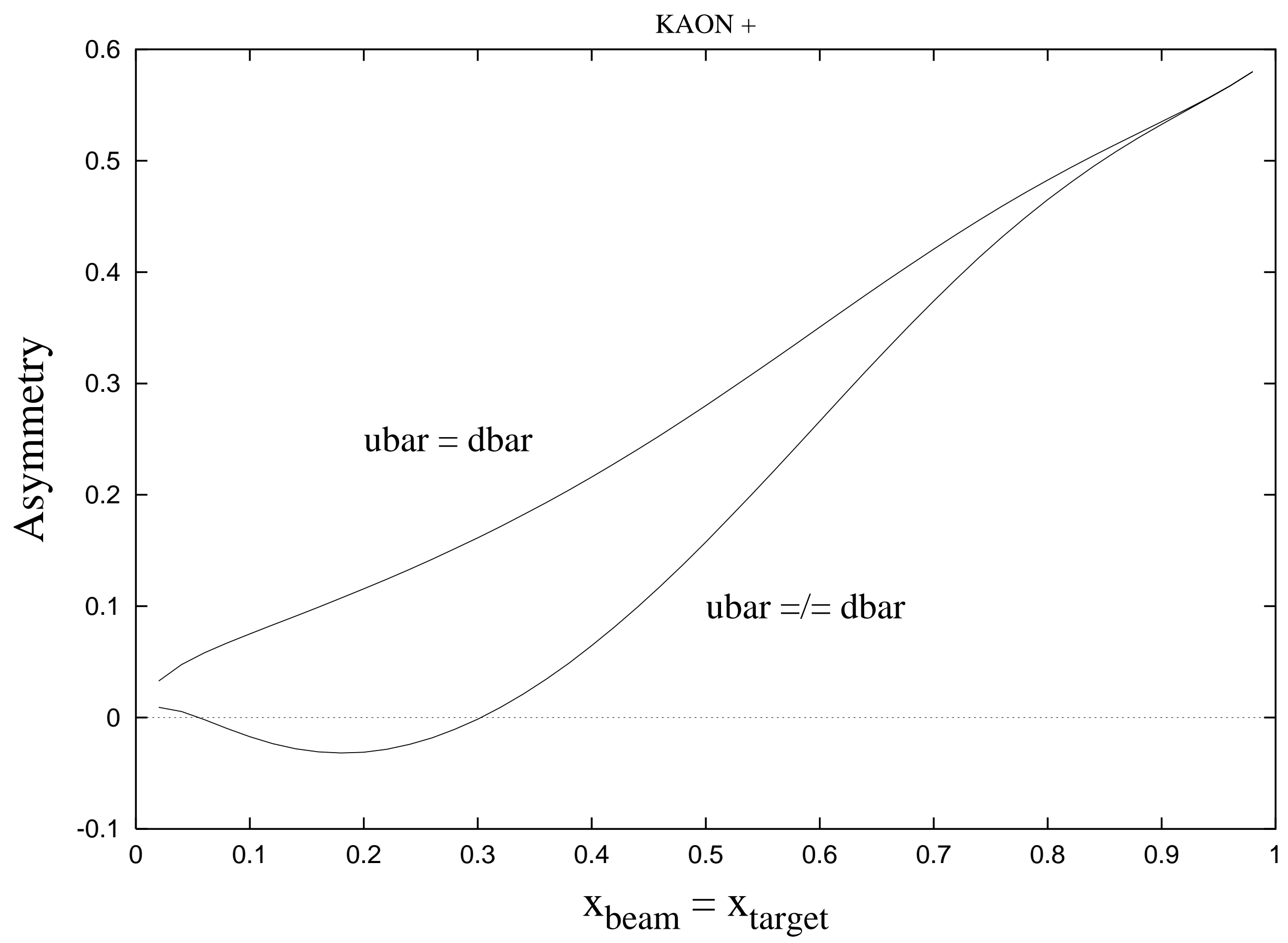




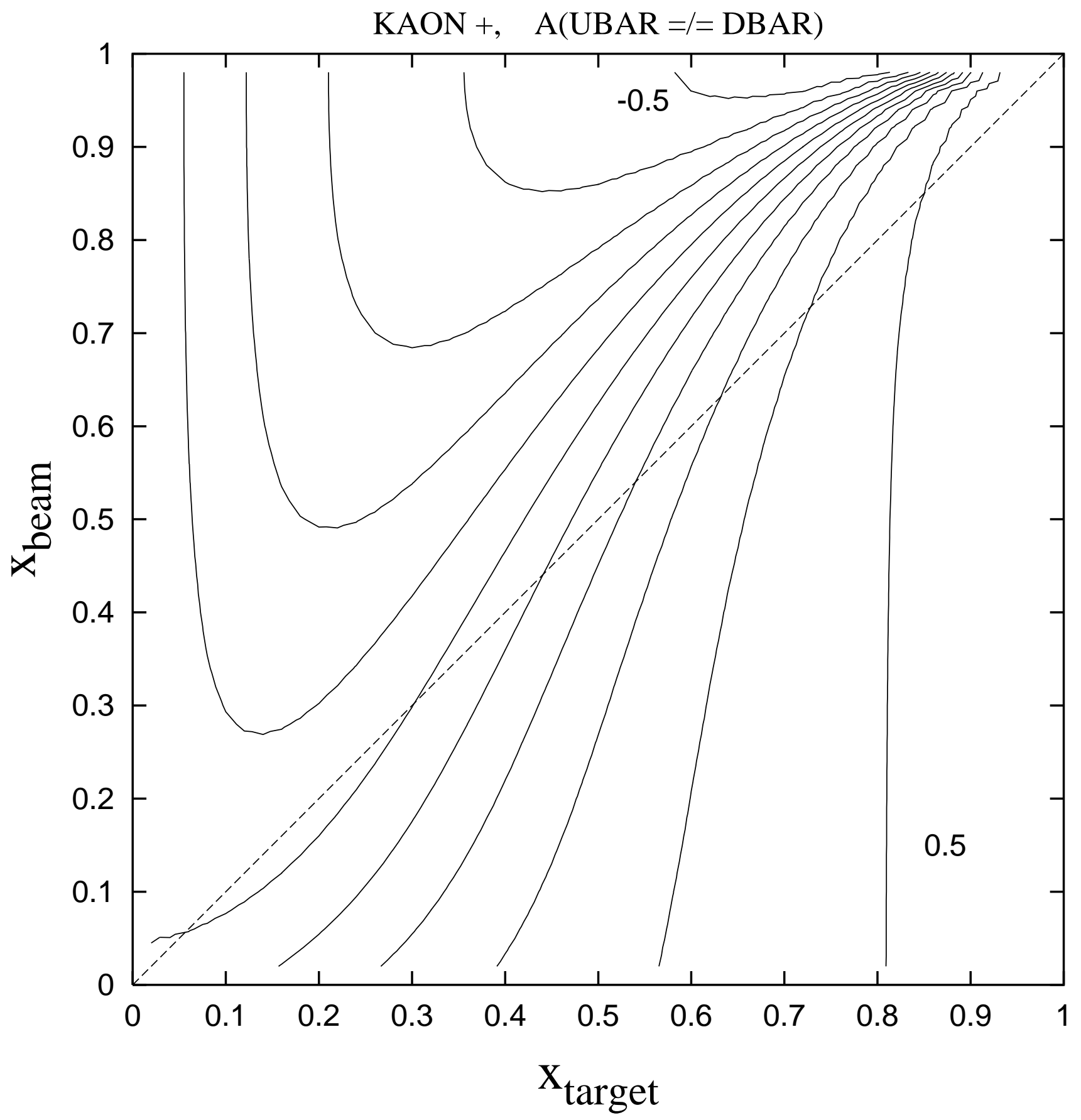




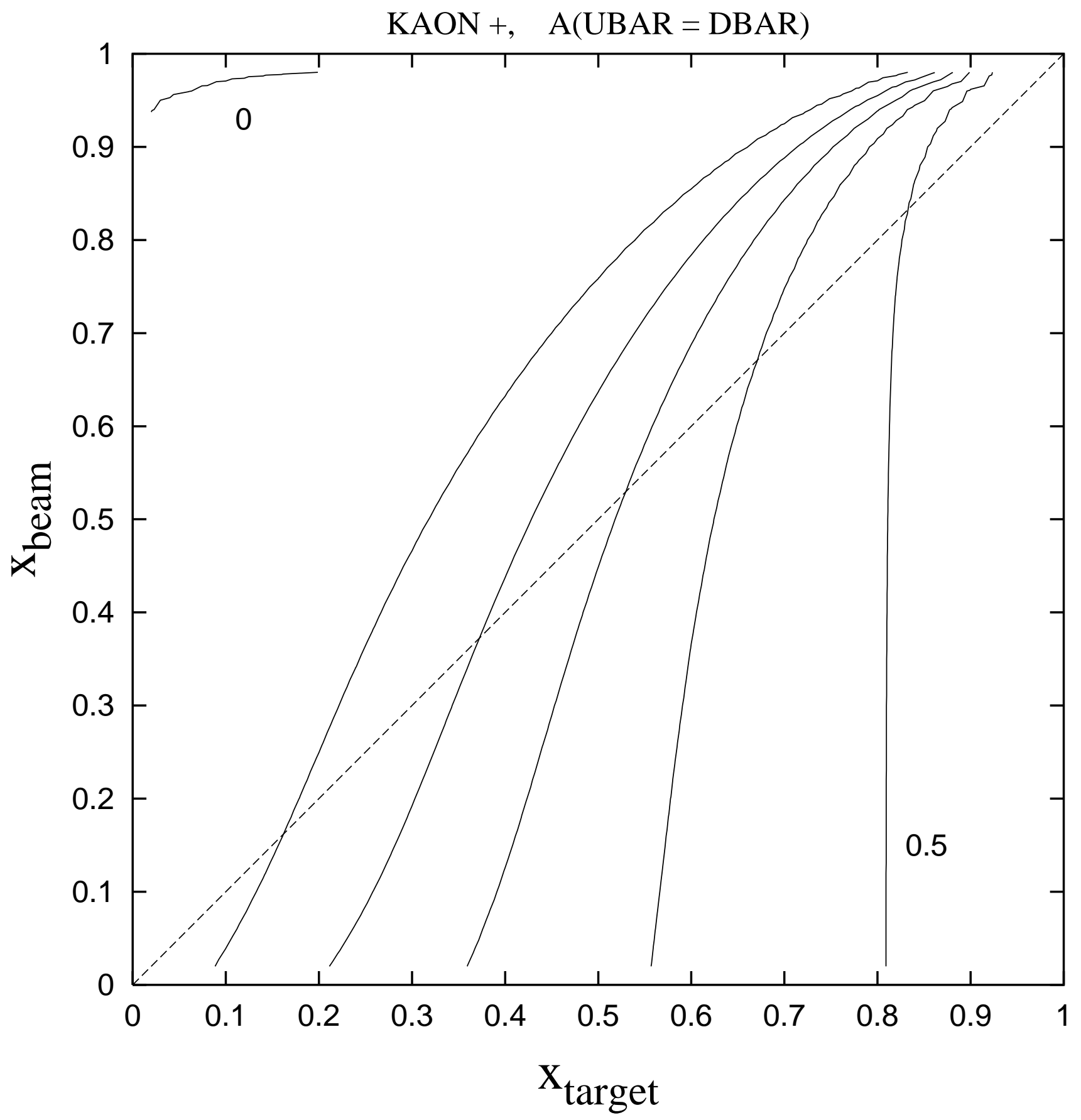




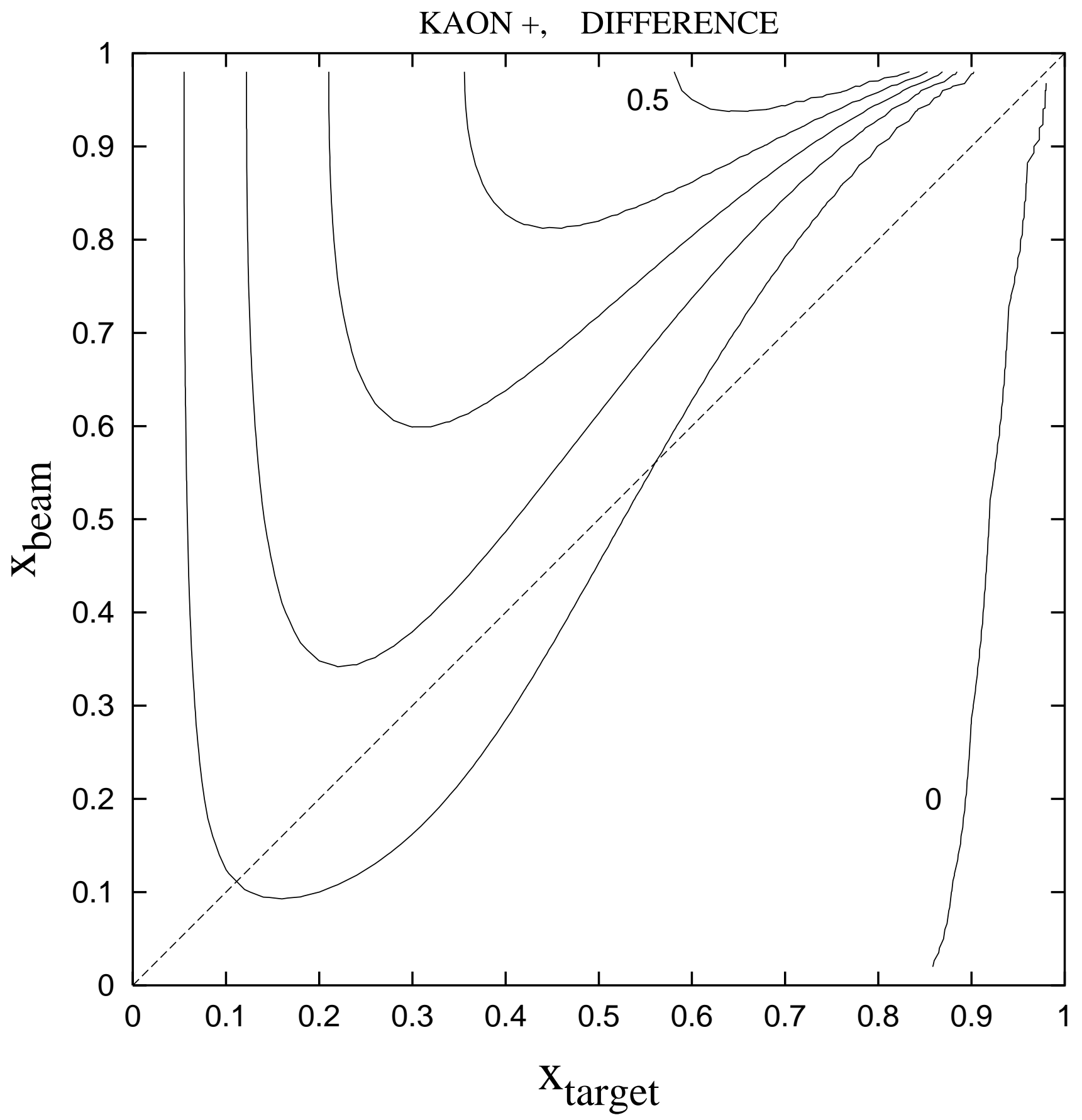

